\title{
ALMA observations of feeding and feedback in nearby Seyfert galaxies: an AGN-driven outflow in NGC 1433 ${ }^{\star}$
}

\author{
F. Combes ${ }^{1}$, S. García-Burillo ${ }^{2}$, V. Casasola ${ }^{3}$, L. Hunt ${ }^{4}$, M. Krips ${ }^{5}$, A. J. Baker ${ }^{6}$, F. Boone ${ }^{7}$, A. Eckart ${ }^{8}$, I. Marquez ${ }^{9}$, \\ R. Neri ${ }^{5}$, E. Schinnerer ${ }^{10}$, and L. J. Tacconi ${ }^{11}$
}

\author{
1 Observatoire de Paris, LERMA, CNRS: UMR8112, 61 Av. de l'Observatoire, 75014 Paris, France \\ e-mail: francoise.combes@obspm.fr \\ 2 Observatorio Astronómico Nacional (OAN)-Observatorio de Madrid, Alfonso XII 3, 28014 Madrid, Spain \\ 3 INAF - Istituto di Radioastronomia \& Italian ALMA Regional Centre, via Gobetti 101, 40129, Bologna, Italy \\ 4 INAF - Osservatorio Astrofisico di Arcetri, Largo E. Fermi 5, 50125 Firenze, Italy \\ 5 IRAM, 300 rue de la Piscine, Domaine Universitaire, 38406 Saint Martin d'Hères, France \\ 6 Dep. of Physics \& Astronomy, Rutgers, the State University of New Jersey, 136 Frelinghuysen road, Piscataway, NJ 08854, USA \\ 7 CNRS, IRAP, 9 Av. colonel Roche, BP 44346, 31028 Toulouse Cedex 4, France \\ 8 I. Physikalisches Institut, Universität zu Köln, Zülpicher Str. 77, 50937 Köln, Germany \\ 9 Instituto de Astrofisica de Andalucía (CSIC), Apdo 3004, 18080 Granada, Spain \\ 10 Max-Planck-Institut für Astronomie (MPIA), Königstuhl 17, 69117 Heidelberg, Germany \\ 11 Max-Planck-Institut für extraterrestrische Physik, Giessenbachstr. 1, Garching bei München, Germany
}

Received 16 July 2013 / Accepted 17 September 2013

\section{ABSTRACT}

\begin{abstract}
We report ALMA observations of CO(3-2) emission in the Seyfert 2 galaxy NGC 1433 at the unprecedented spatial resolution of $0.5=24 \mathrm{pc}$. Our aim is to probe active galactic nucleus (AGN) feeding and feedback phenomena through the morphology and dynamics of the gas inside the central kpc. The galaxy NGC 1433 is a strongly barred spiral with three resonant rings: one at the ultraharmonic resonance near corotation, and the others at the outer and inner Lindblad resonances (OLR and ILR). A nuclear bar of $400 \mathrm{pc}$ radius is embedded in the large-scale primary bar. The CO map, which covers the whole nuclear region (nuclear bar and ring), reveals a nuclear gaseous spiral structure, inside the nuclear ring encircling the nuclear stellar bar. This gaseous spiral is well correlated with the dusty spiral seen in Hubble Space Telescope (HST) images. The nuclear spiral winds up in a pseudo-ring at $\sim 200 \mathrm{pc}$ radius, which might correspond to the inner ILR. Continuum emission is detected at $0.87 \mathrm{~mm}$ only at the very centre, and its origin is more likely thermal dust emission than non-thermal emission from the AGN. It might correspond to the molecular torus expected to exist in this Seyfert 2 galaxy. The $\mathrm{HCN}(4-3)$ and $\mathrm{HCO}^{+}(4-3)$ lines were observed simultaneously, but only upper limits are derived, with a ratio to the $\mathrm{CO}(3-2)$ line lower than $1 / 60$ at $3 \sigma$, indicating a relatively low abundance of very dense gas. The kinematics of the gas over the nuclear disk reveal rather regular rotation only slightly perturbed by streaming motions due to the spiral; the primary and secondary bars are too closely aligned with the galaxy major or minor axis to leave a signature in the projected velocities. Near the nucleus, there is an intense high-velocity $\mathrm{CO}$ emission feature redshifted to $200 \mathrm{~km} \mathrm{~s}^{-1}$ (if located in the plane), with a blue-shifted counterpart, at $2^{\prime \prime}(100 \mathrm{pc})$ from the centre. While the CO spectra are quite narrow in the centre, this wide component is interpreted as an outflow involving a molecular mass of $3.6 \times 10^{6} M_{\odot}$ and a flow rate $\sim 7 M_{\odot} / y r$. The flow could be in part driven by the central star formation, but is mainly boosted by the AGN through its radio jets.
\end{abstract}

Key words. galaxies: active - galaxies: individual: NGC 1433 - galaxies: ISM - galaxies: kinematics and dynamics galaxies: nuclei - galaxies: spiral

\section{Introduction}

It is now observationally well established that supermassive black holes (SMBHs) reside in the nuclei of all galaxies with massive spheroids in the Local Universe and at higher redshifts as well (e.g. Kormendy \& Ho 2013). Quasars at high redshift and Seyfert nuclei locally are fueled by accretion of material onto the SMBH. Although much progress has been made on both theoretical and observational fronts in the last decade, the relationship of black hole growth with galaxy formation and evolution is still far from being completely understood.

One of the outstanding problems is to identify the mechanism that drives gas from the disk towards the nucleus, removing its large angular momentum, to feed the central black hole and trigger the nuclear activity. Theoretically, broad-brush

\footnotetext{
* Based on observations carried out with ALMA in Cycle 0.
}

solutions have been found; cosmological simulations rely on merger-driven gas inflow via bar instabilities to feed a central starburst and fuel the SMBH (e.g., Hopkins et al. 2006; di Matteo et al. 2008). Nevertheless, in the Local Universe, no clear correlation has been found between the presence of an active galactic nucleus (AGN) and either companions or the presence of bars (see e.g. Combes 2003, 2006; Jogee 2006, for reviews). It is possible that locally the relation between these large-scale phenomena and the duty cycle of nuclear fueling is masked by different timescales. Indeed, the presence of resonant rings, vestiges of a previous bar, appears to be correlated with Seyfert activity (Hunt \& Malkan 1999). It could also be that gas inflow is not always possible because of dynamical barriers (e.g., nuclear rings, see Piner et al. 1995; Regan \& Teuben 2004).

To assess potential inhibitors of the ubiquitous gas inflow assumed in simulations, we must examine the nuclear kinematics around local AGN. This can be best done with molecular tracers, 
since in galaxy centres HI is typically converted to molecular gas; $\mathrm{CO}$ line emission is therefore our best probe, and in particular $\mathrm{CO}(3-2)$, which traces the high density gas $\left(10^{4}-10^{5} \mathrm{~cm}^{-3}\right)$ in the dense AGN circumnuclear regions (as we have shown in Boone et al. 2011). The $\mathrm{HCN}$ and $\mathrm{HCO}^{+}$line emission should trace the densest material (at least $10^{7} \mathrm{~cm}^{-3}$ ), and diagnose its excitation and chemistry. We have undertaken during the last decade the NUclei of GAlaxies (NUGA) program to study the gas distributions in nearby AGN, and find clues to their fueling. In the dozen nearby Seyfert or LINER galaxies observed with the IRAM Plateau de Bure interferometer (PdBI) in $\mathrm{CO}(2-1)$, we achieved a spatial resolution of $50-100 \mathrm{pc}$, and frequently worse for the most distant galaxies. In these galaxies, a large variety of gas distributions have been found; however, we detected on-going AGN feeding at $0.1-1 \mathrm{kpc}$ scales for only five out of twelve cases: NGC 2782 (Hunt et al. 2008, bar triggered by an interaction), NGC 3147 (Casasola et al. 2008), NGC 3627 (Casasola et al. 2011), NGC 4579 (Garcìa-Burillo et al. 2009), and NGC 6574 (Lindt-Krieg et al. 2008). The most common feeding mechanism in these galaxies appears to be kinematically decoupled embedded bars, i.e. the combination of a slowly rotating kpc-scale stellar bar (or oval) and a kinematically decoupled nuclear bar, with overlapping dynamical resonances. Such resonances and kinematic decoupling are fostered by a large central mass concentration and high gas fraction. The gas is first stalled in a nuclear ring (a few $100 \mathrm{pc}$ scale), and then driven inward under the influence of the decoupled nuclear bar. However, because of insufficient resolution, our previous observations were unable to probe the gas within $100 \mathrm{pc}$ of the AGN most of the time.

In this paper, we present ALMA Cycle 0 observations in the $\mathrm{CO}(3-2)$ line of the Seyfert 2 NGC 1433, where the beam is $24 \mathrm{pc}$ in size. The nearby distance $(9.9 \mathrm{Mpc})$ and low inclination of $33^{\circ}$ make NGC 1433 an ideal target to test and refine the scenario of AGN feeding and feedback, and to constrain $\mathrm{BH}$ models which are only now beginning to examine in detail gas structures within 100 pc (Hopkins \& Quataert 2010; Perez-Beaupuits et al. 2011).

Up to now, resolution at scales of tens of pc has been obtained only in a few Seyfert galaxies, and only in hot or warm gas tracers. The best example is NGC 1068, the closest Seyfert 2 prototype, where near-IR $\mathrm{H}_{2}$ lines have been mapped with SINFONI at 0.'075 resolution $(5.2 \mathrm{pc})$ by Müller-Sanchez et al. (2009). The behaviour of this hot (1000-2000 K) gas is not yet settled, however; while an outflow model is proposed by Galliano \& Alloin (2002), and a warped disk model by Schinnerer et al. (2000), Müller-Sanchez et al. (2009) propose a strong inflow model. Krips et al. (2011) explain their SMA $\mathrm{CO}(3-2)$ map at $0{ }^{\prime} 6=40 \mathrm{pc}$ resolution by a rotating disk plus an outflow of the disk gas due to shocks and/or a circumnuclear disk-jet interaction. Thus, gas inflow could fuel the AGN at a 10 -pc scale, and the jet-gas interaction could simultaneously drag gas outwards on scales of hundreds of pc. It has also been suggested that the presence of an outflow in the circumnuclear disk of NGC 1068 is responsible for the large-scale molecular shocks revealed by strong $\mathrm{SiO}$ emission in this galaxy (GarcìBurillo et al. 2010). The outflow is clearly seen in ALMA data (Garcìa-Burillo et al., in prep.). Typical outflow velocities are found of the order of $200 \mathrm{~km} \mathrm{~s}^{-1}$ in NGC 1068 .

An important ingredient in cosmological simulations is feedback, which can regulate SMBH growth and suppress star formation (e.g., Croton et al. 2006; di Matteo et al. 2008, and references therein). Molecular observations can constrain specific feedback mechanisms by discovering molecular outflows through their high-velocity wings, and can determine their origin (star formation or AGN) through high resolution observations. Chung et al. (2011) showed the ubiquitous presence of $1000 \mathrm{~km} \mathrm{~s}^{-1}$ molecular outflows in starbursts with star formation rates (SFRs) larger than $100 M_{\odot} / \mathrm{yr}$ (see also Feruglio et al. 2010; Fischer et al. 2010; Sturm et al. 2011). The CO emission in the high-velocity wings may generally represent $25 \%$ of the total observed emission. In NGC 1068 the outflow, if present, is only of the order of $200 \mathrm{~km} \mathrm{~s}^{-1}$ and is entrained by the radio jet. Coil et al. (2011) also find that galactic winds are frequent in ionized gas lines in post-starburst and AGN host galaxies at $0.2<z<0.8$, but they are low-velocity winds, likely due to supernovae. High-velocity winds, driven by an AGN, might be frequent in molecular gas (Leon et al. 2007; Feruglio et al. 2010, 2013; Alatalo et al. 2011; Nesvadba et al. 2011; Dasyra \& Combes 2012; Aalto et al. 2012; Spoon et al. 2013; Veilleux et al. 2013), as well as in the ionized or atomic gas component (Rupke et al. 2005; Riffel \& Storchi-Bergmann 2011). In Arp 220, Sakamoto et al. (2009) have discovered 100-200 $\mathrm{km} \mathrm{s}^{-1}$ outflows through P-Cygni profiles in $\mathrm{HCO}^{+}(3-2), \mathrm{HCO}^{+}(4-3)$, and $\mathrm{CO}(3-2)$ along the line of sight to the nucleus. They interpret this gas as driven outwards by the nuclear starburst. Because NGC 1433 is not an IR-luminous starburst, it is unlikely that an AGN wind close to the nucleus would be swamped by a starburst wind, thus facilitating its identification with ALMA's high resolution.

\subsection{NGC 1433}

The system NGC 1433 is a nearby active barred galaxy; it is a member of the Dorado group which includes 26 galaxies (Kilborn et al. 2005). We selected it from a sample of lowluminosity AGN spirals already detected in $\mathrm{CO}$ emission for its proximity and moderate inclination. It was classified as Seyfert 2 by Veron-Cetty \& Veron (1986), because of its strong nuclear emission lines and its high $[\mathrm{NII}] / \mathrm{H} \alpha$ ratio. However, Sosa-Brito et al. (2001) prefered to classify it as LINER, because of its $[\mathrm{OIII}] / \mathrm{H} \beta$ ratio, which is just at the limit between Seyfert and LINERs. Liu \& Bregman (2005) detected the nuclear point source in X-rays with ROSAT.

The galaxy has a rich network of dusty filaments around the nucleus. Its morphology reveals conspicuous rings (Buta 1986; Buta et al. 2001); the presence of nuclear, inner, and outer rings is the motivation of its nickname of the "Lord of Rings" (Buta \& Combes 1996). Table 2 presents the sizes and orientations of the main dynamical features. Near-IR images have revealed a nuclear bar inside the nuclear ring, of radius $\sim 400$ pc (Jungwiert et al. 1997). The ring is the site of a starburst and is patchy in UV (continuum HST image from Maoz et al. 1996). Thirty one compact sources contribute $12 \%$ of the UV light. Inside the ring the dust traces a flocculent or multiple-arm nuclear spiral structure (HST image from Peeples \& Martini 2006). There is a peak of $6 \mathrm{mJy}$ in radio continuum emission at $843 \mathrm{MHz}$ in the centre (Harnett 1987), with a weak extension along the bar. The HI $21 \mathrm{~cm}$ emission map (Ryder et al. 1996) reveals that the atomic gas is concentrated in the inner and outer rings, with some depletion in the nuclear ring and bar region. In contrast, the central region is filled with molecular hydrogen (Bajaja et al. 1995, CO SEST map). Our ALMA single pointing includes in its field of view (FOV) all the nuclear bar and nuclear spiral gas.

\section{Observations}

The observations were carried out with the Atacama Large Millimeter/submillimeter Array (ALMA) telescope in Cycle 0, 


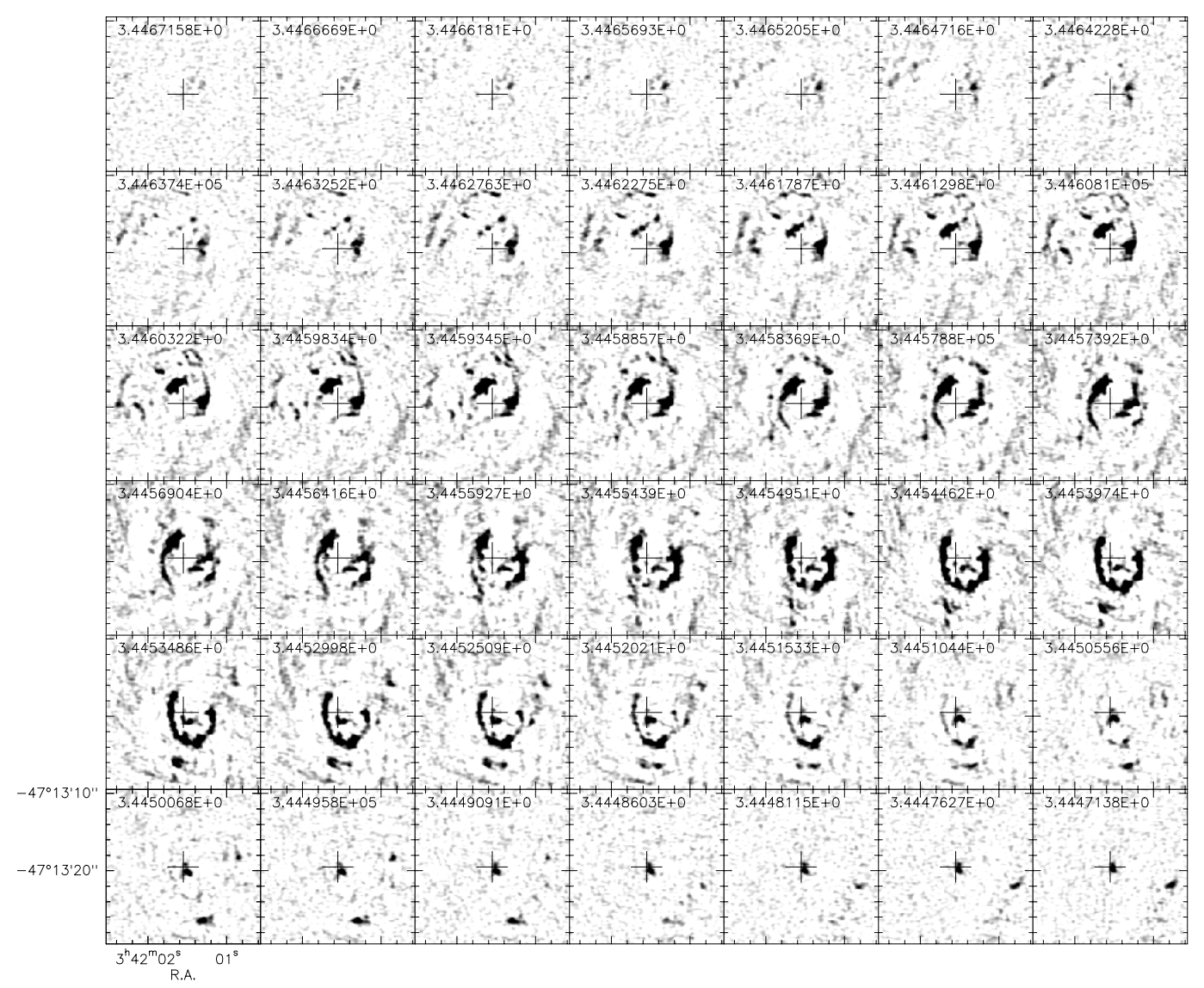

Fig. 1. Channel maps of $\mathrm{CO}(3-2)$ emission in the centre of NGC 1433. Each of the 42 square boxes is $20^{\prime \prime}$ in size, while the primary beam is $18^{\prime \prime}$ in diameter. Channels are separated by $4.24 \mathrm{~km} \mathrm{~s}^{-1}$. They are plotted from 978 (top left) to $1152 \mathrm{~km} \mathrm{~s}^{-1}$ (bottom right, the panels are labelled in frequency). The synthesized beam is $0{ }^{\prime} 56 \times 00^{\prime} 42\left(\mathrm{PA}=85^{\circ}\right)$. The centre of the maps is the phase centre of the interferometric observations given in Table 1. The colour scale is linear, between 1 and $30 \mathrm{mJy} / \mathrm{beam}$.

with 19 antennae, during June and July 2012. The galaxy NGC 1433 was observed simultaneously in $\mathrm{CO}(3-2), \mathrm{HCO}^{+}(4-$ $3), \mathrm{HCN}(4-3)$, and continuum, with Band 7. The sky frequencies were $344.56 \mathrm{GHz}, 355.46 \mathrm{GHz}, 353.24 \mathrm{GHz}$, and $343.27 \mathrm{GHz}$, respectively. The observations were done in three blocks, with a total duration of two hours. For each period, NGC 1433 was observed for $27 \mathrm{~min}$; the median system temperatures were $T_{\text {sys }}=140,230$, and $160 \mathrm{~K}$.

The observations were centred on the nucleus, with a single pointing covering a FOV of $18^{\prime \prime}$. The Cycle 0 extended configuration provides in Band 7 a beam of $0.56 \times 0 . ' 42$, with a $\mathrm{PA}$ of $85^{\circ}$. The galaxy was observed in dual polarization mode with $1.875 \mathrm{GHz}$ total bandwidth per baseband, and a velocity resolution of $0.488 \mathrm{MHz} \sim 0.42 \mathrm{~km} \mathrm{~s}^{-1}$. The spectra were then smoothed to $4.88 \mathrm{MHz}\left(4.24 \mathrm{~km} \mathrm{~s}^{-1}\right)$ to build channel maps.

This choice of correlator configuration, selected to simultaneously observe three lines, provided a velocity range of $1600 \mathrm{~km} \mathrm{~s}^{-1}$ for each line, but did not centre the lines (200 $\mathrm{km} \mathrm{s}^{-1}$ on one side and $1400 \mathrm{~km} \mathrm{~s}^{-1}$ on the other) which is adequate for a nearly face-on galaxy, and $1800 \mathrm{MHz}$ bandwidth in the continuum. The total integration time provided an $\mathrm{rms}$ of $0.09 \mathrm{mJy} /$ beam in the continuum, and $\sim 3 \mathrm{mJy} /$ beam in the line channel maps (corresponding to $\sim 170 \mathrm{mK}$, at the obtained spatial resolution). The flux calibration was done with the nearby quasar J0334-401, which is regularly monitored at ALMA, and resulted in $10 \%$ accuracy.

The data were calibrated, and cleaned using first a mask at the $50 \mathrm{mJy}$ emission level, and then the $30 \mathrm{mJy}$ level. The final cube is $360 \times 360$ pixels with 0 .' 1 per pixel in the plane of the sky, and has 60 channels of $4.24 \mathrm{~km} \mathrm{~s}^{-1}$ width. The data were calibrated, imaged, and cleaned with the CASA software (v3.3; McMullin et al. 2007), and the analysis was then finalized with the GILDAS software (Guilloteau \& Lucas 2000).

The final maps were corrected for primary beam attenuation to compute fluxes, but were kept uncorrected for the plots. Almost no $\mathrm{CO}(3-2)$ emission was detected outside the full-width half-power (FWHP) primary beam. Because of missing short spacings, extended emission was filtered out at scales larger than $\sim 3^{\prime \prime}$ in each channel map. The elongated features corresponding to the dust lanes that were detected along the arms and rings are, however, quite narrow (thinner than $2^{\prime \prime}$ as in HST images), so the missing-flux problem might not be severe in individual velocity slices. Low-level negative sidelobes adjacent to bright emission were observed.

\section{Results}

Figure 1 displays 42 of the $\mathrm{CO}(3-2)$ channel maps, with a velocity range of $175 \mathrm{~km} \mathrm{~s}^{-1}$ and a velocity resolution of $4.24 \mathrm{~km} \mathrm{~s}^{-1}$. The velocity field is regular, although perturbed by the tightlywound spiral structure (see also Fig. 2). At the outermost channels, the emission at the highest velocities does not occur primarily at large radii, but mainly towards the centre.

\subsection{Molecular gas distribution and morphology}

To measure fluxes we used a clipped cube where all pixel values $<2 \sigma(6 \mathrm{mJy} /$ beam $)$ were set to zero. The mean intensity is 


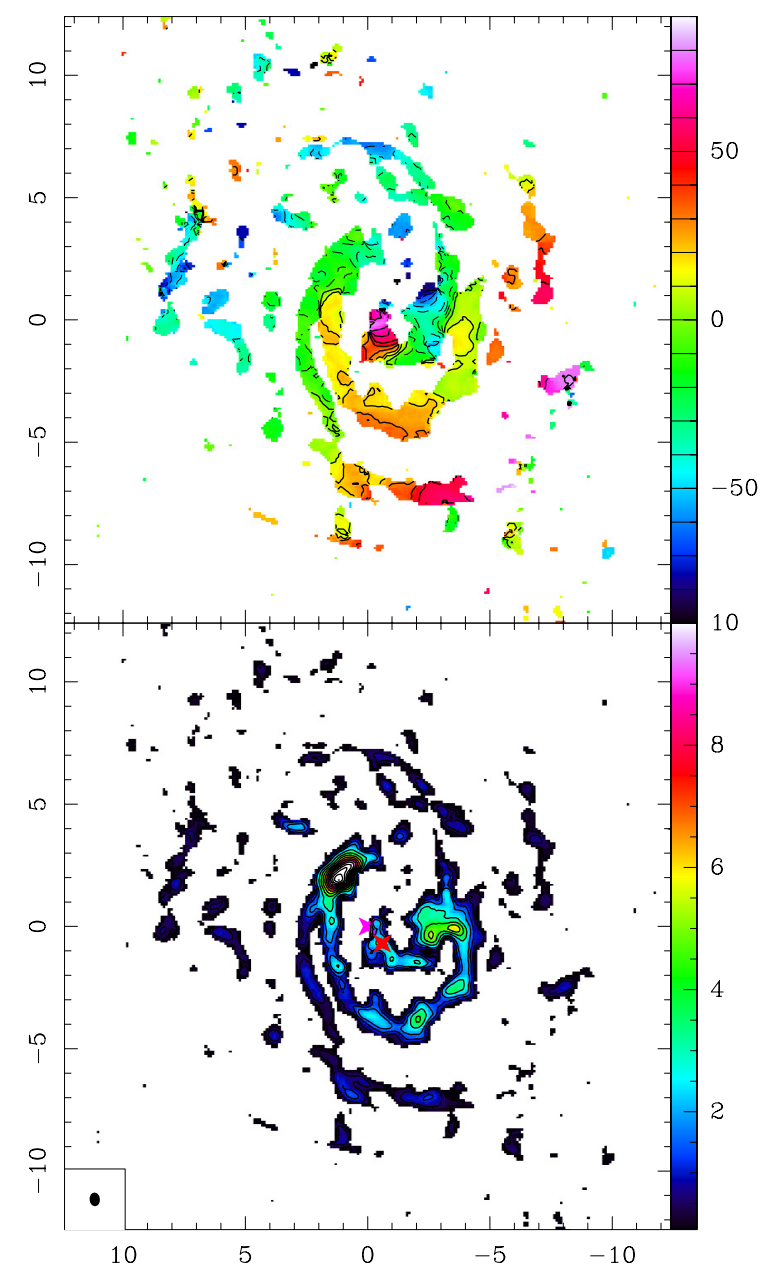

Fig. 2. Velocity field (top) and integrated intensity (bottom) of the $\mathrm{CO}(3-2)$ emission in the centre of NGC 1433. Coordinates are RA-Dec in arcsec relative to the phase centre (see Table 1). The colour scale ranges are in $\mathrm{km} \mathrm{s}^{-1}$ relative to $1075 \mathrm{~km} \mathrm{~s}^{-1}$ (top) and in Jy/beam.MHz (or $0.87 \mathrm{Jy} /$ beam $\mathrm{km} \mathrm{s}^{-1}$ ) at the bottom. The beam size of $0{ }^{\prime} 56 \times 0.0^{\prime} 42$ is indicated at the bottom left. The phase centre is a pink cross, and the new adopted centre is the red cross, shown in the bottom panel.

plotted in Fig. 2 (bottom). Since the galaxy is more extended than the primary beam, it is difficult to quantify the missing flux. We compare it to the central spectrum obtained with a single dish in Sect. 3.4; however, these observations were obtained with the SEST in CO(1-0) with a 43" beam. Nevertheless, our FOV encompasses the entire nuclear ring, and the emission in this nuclear region has by far the strongest surface density at many wavelengths (Buta et al. 2001; Comeron et al. 2010; Ho et al. 2011).

We superposed the CO map onto the HST maps in the $B$, $V$, and $I$ filters. All show a remarkable similarity in morphology, as displayed in Fig. 3. The features are so distinct that they were used to align the HST images which suffered from an inexact astrometry. The $\mathrm{CO}$ emission nicely corresponds to the dust lanes, interleaved with the bright regions. The gas and the dust are closely mixed, and reveal a multi-arm structure with a low pitch angle. There is not a well-defined density-wave here, but rather a more flocculent spiral structure with multiple branches. The structure is easily appreciated, thanks to the low inclination of the galaxy $\left(33^{\circ}\right.$, Table 1$)$. This spiral structure is entirely included inside the nuclear ring of $\sim 10^{\prime \prime}=0.5 \mathrm{kpc}$ in radius (Buta et al. 2001, see Table 2).
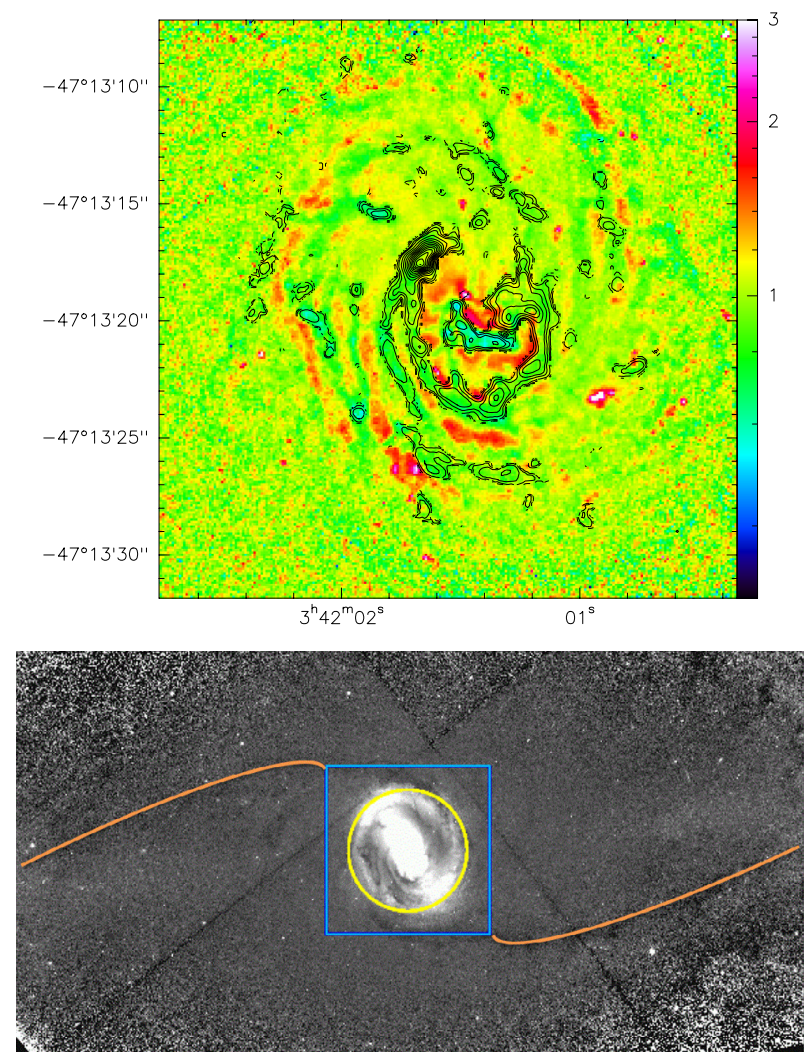

Fig. 3. Top: overlay of $\mathrm{CO}(3-2)$ contours on the unsharp-masked blue (F450W) HST image. The HST image has been aligned to correspond to the ALMA astrometry. Bottom: unsharp masking of the HST I-image of NGC 1433, covering the nuclear ring and the dust lanes along the primary bar. The FWHP of the primary beam is indicated in yellow ( $18^{\prime \prime}$ in diameter), and the FOV of the CO map in Fig. 2 and in the above image is indicated in blue (square of $24{ }^{\prime \prime} 8$ on a side). The characteristic dust lanes on the leading edge of the main bar are outlined in orange.

What is remarkable is the large difference between the gas complex morphology in this nuclear region, revealed by ALMA and the already known smoother stellar morphology (Buta et al. 2001). The lower panel of Fig. 3 shows an unsharp-masked red image of the nuclear region, embedded in the primary bar whose leading dust lanes are marked. As is frequently found in strong primary bars of early-type spirals, the dust lanes wind up onto the nuclear ring, which corresponds to the inner Lindblad resonance, and a secondary nuclear bar decouples inside (e.g. Buta $\&$ Combes 1996). However, the gas does not follow the stellar nuclear ring, but instead flows through a flocculent spiral onto an even smaller nuclear ring of $\sim 200 \mathrm{pc}$ radius, and from there reaches the very centre, at least at the $20 \mathrm{pc}$ scale, our resolution.

The molecular gas morphology reveals notable asymmetries; for instance, the peak of $\mathrm{CO}$ emission is not in the centre but in a NE cloud complex, at about 4 " from the centre (200 pc), with no SW counterpart. In the very centre, the emission extends $2^{\prime \prime}$ to the SW, but with a corresponding hole in the NE. This might indicate an $m=1$ Fourier component in addition to the $m=2$ and $m=3$ arm features. To determine whether one particular $m$ component dominates, we have computed the Fourier decomposition of the 2D gas density once the galaxy disk has been deprojected to the sky plane ${ }^{1}$. Figure 4 displays the face-on molecular gas distribution. The pseudo-ring at radius $\sim 4^{\prime \prime}$ corresponding

1 The decomposition is performed using the new centre defined in Sect. 3.2. 
Table 1. Basic data for the NGC 1433 galaxy.

\begin{tabular}{lcc}
\hline \hline Parameter & Value $^{b}$ & Reference $^{c}$ \\
\hline$\alpha_{\mathrm{J} 2000}{ }^{a}$ & $03^{\mathrm{h}} 42^{\mathrm{m}} 01.55^{\mathrm{s}}$ & $(1)$ \\
$\delta_{\mathrm{J} 2000}{ }^{a}$ & $-47^{\circ} 13^{\prime} 19.5^{\prime \prime}$ & $(1)$ \\
$V_{\text {hel }}$ & $1075 \mathrm{~km} \mathrm{~s}^{-1}$ & $(1)$ \\
RC3 type & $\left(\mathrm{R}^{\prime}\right) \mathrm{SB}(\mathrm{r}) \mathrm{ab}$ & $(1)$ \\
Nuclear activity & Seyfert 2 & $(2)$ \\
Inclination & $333^{\circ} 0$ & $(3)$ \\
Position angle & $199^{\circ} \pm 1^{\circ}$ & $(3)$ \\
Distance & $9.9 \mathrm{Mpc}\left(1^{\prime \prime}=48 \mathrm{pc}\right)$ & $(4)$ \\
$L_{B}$ & $1.0 \times 10^{10} L_{\odot}$ & $(4)$ \\
$M_{\mathrm{HI}}$ & $5.5 \times 10^{8} M_{\odot}$ & $(5)$ \\
$M_{\mathrm{H}}$ & $2.3 \times 10^{8} M_{\odot}$ & $(6)$ \\
$M_{\mathrm{dust}}(60$ and $100 \mu \mathrm{m})$ & $2.5 \times 10^{6} M_{\odot}$ & $(7)$ \\
$L_{\mathrm{FIR}}$ & $1.3 \times 10^{9} L_{\odot}$ & $(7)$ \\
$\alpha_{\mathrm{J} 2000}{ }^{d}$ & $03^{\mathrm{h}} 42^{\mathrm{m}} 01.49^{\mathrm{s}}$ & New centre \\
$\delta_{\mathrm{J} 2000}{ }^{\circ}$ & $-47^{\circ} 13^{\prime} 20.2^{\prime \prime}$ & New centre \\
\hline
\end{tabular}

Notes. ${ }^{(a)}\left(\alpha_{\mathrm{J} 2000}, \delta_{\mathrm{J} 2000}\right)$ is the phase tracking centre of our ${ }^{12} \mathrm{CO}$ interferometric observations. ${ }^{(b)}$ Luminosity and mass values extracted from the literature have been scaled to the distance of $D=9.9 \mathrm{Mpc}$. (c) (1) NASA/IPAC Extragalactic Database (NED, http://nedwww . ipac.caltech.edu/); (2) Veron-Cetty \& Veron (1986); (3) Buta et al. (2001); (4) HyperLeda; (5) Ryder et al. (1996); (6) Bajaja et al. (1995), reduced to the conversion factor $2.3 \times 10^{20} \mathrm{~cm}^{-2} /\left(\mathrm{K} \mathrm{km} \mathrm{s}^{-1}\right)$; (7) IRAS Catalog. ${ }^{(d)}$ New adopted centre, coinciding with the continuum peak.

Table 2. Main dynamical features in NGC 1433.

\begin{tabular}{lcc}
\hline \hline Feature & Radius & $\mathrm{PA}\left(^{\circ}\right)$ \\
\hline Nuclear bar & $9^{\prime \prime}(430 \mathrm{pc})$ & 31 \\
Nuclear ring & $9.5^{\prime \prime}(460 \mathrm{pc})$ & 31 \\
Primary bar & $83^{\prime \prime}(4 \mathrm{kpc})$ & 94 \\
Inner ring & $108^{\prime \prime}(5.2 \mathrm{kpc})$ & 95 \\
Outer ring & $190^{\prime \prime}(9.1 \mathrm{kpc})$ & 15 \\
\hline
\end{tabular}

to $200 \mathrm{pc}$ is clearly visible and nearly round. We have computed the radial distribution of the various Fourier components, normalized to the axi-symmetric power. The surface density of the gas has been decomposed as

$\Sigma(r, \phi)=\Sigma_{0}(r)+\sum_{m} a_{m}(r) \cos \left(m \phi-\phi_{m}(r)\right)$

and the amplitude of the various Fourier components $m$ are normalized as $A_{m}(r)=a_{m}(r) / \Sigma_{0}(r)$. As a result, all $A_{m}(r)$ coefficients show noisy behaviour, at a maxium amplitude of 0.5 , but there is no particular dominance of any $m$ feature.

\subsection{Continuum emission}

Besides the $\mathrm{CO}(3-2)$ line, continuum emission was detected at $0.87 \mathrm{~mm}$. For that, the fourth band of width $468.8 \mathrm{MHz}$ was used, with a rms noise level of $0.15 \mathrm{mJy}$. Figure 5 displays the $\mathrm{CO}(3-2)$ contours superposed onto the continuum map. The peak emission is just detected at $3 \sigma$, about $0.5 \mathrm{mJy}$. The emission is extended in the east-west direction; its size is $1^{\prime \prime} \times 0.0^{\prime} 5$.

\subsubsection{Recentring}

To establish the origin of the continuum emission, one issue is to determine the exact position of the AGN. We observed with a phase centre corresponding to the peak of the near-IR emission

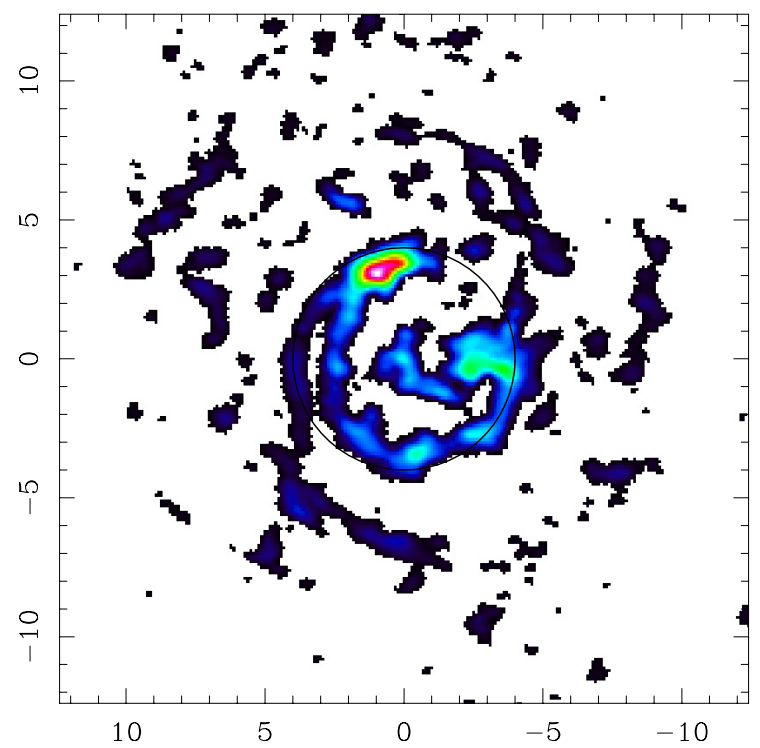

Fig. 4. Deprojection of the CO emission towards a face-on disk, centred on the new adopted centre given in Table 1 . The pseudo-ring of $4^{\prime \prime}$ radius, here highlighted with a black circle, is standing out, nearly round.

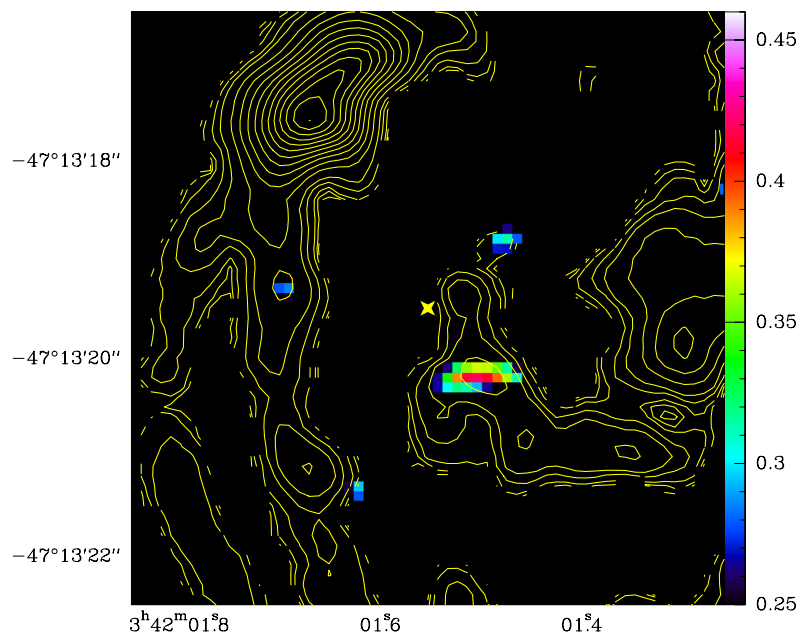

Fig. 5. Overlay of $\mathrm{CO}(3-2)$ contours on the $0.87 \mathrm{~mm}$ continuum image. The FOV is $6^{\prime \prime}$ in diameter. The yellow star shows the phase centre given in Table 1, while the peak of the continuum is our new adopted centre. The colour palette unit is mJy.

of the stellar component, which is known only within 0.7 uncertainty (e.g. 2MASS catalog, 2003). The HST maps, in $B, V$, and $I$ would be precise enough, but they are all affected by dust obscuration. In particular there is a conspicuous dust-lane extending nearly horizontally in the SW. The continuum emission peaks at a position $\left(-0 .{ }^{\prime} 6,-0.7\right)$ with respect to our phase centre, so perfectly compatible within the uncertainty. This position is, however, better centred with respect to the $\mathrm{CO}$ emission. We therefore choose to adopt the peak of the continuum emission as the new centre. This is also perfectly compatible with the position of the X-ray nuclear point source seen by Liu \& Bregman (2005). Although the AGN might not correspond exactly to the peak of the stellar component, it is possible that our new centre is also the correct position of the AGN and the supermassive black hole. However it is unclear whether or not the $0.87 \mathrm{~mm}$ AGN synchrotron emission is detected. 


\subsubsection{Slope of radio-continuum emission}

Radio continuum emission has been detected at $35 \mathrm{~cm}$ by Harnett (1987) with a resolution of $43^{\prime \prime} \times 58^{\prime \prime}$; the emission is extended, and shows $6 \mathrm{mJy}$ in the central beam. The galaxy NGC 1433 has also been observed at $21 \mathrm{~cm}$ with ATCA by Ryder et al. (1996), with a spatial resolution of $30^{\prime \prime}$. The central emission is $3.4 \mathrm{mJy}$, quite similar to what is obtained at the ends of the bar from the HII regions. Since the whole nuclear region is included in their central beam, it is possible that all the radio emission comes from star formation in the ring or nuclear region (both synchrotron from supernovae, and free-free emission). The continuum becomes $2 \mathrm{mJy}$ at $4.8 \mathrm{GHz}$, with no polarisation (Stil et al. 2009). Comparing the central fluxes at $21 \mathrm{~cm}$ and $0.87 \mathrm{~mm}$, the slope of the radio spectrum would be -0.35 , which could be a mixture of synchrotron with a steeper spectrum $(-0.7)$, and freefree emission with slope -0.1 . Both steep radio spectra (Sadler et al. 1995), and flat ones (Ulvestad \& Ho 2001) have been found in Seyfert spiral galaxies, so it is not possible to conclude on the AGN contribution in the centre. From the $\mathrm{H} \alpha$ flux it would be possible in principle to estimate the fraction of free-free emission expected in the centre, but the spatial resolution $\left(2^{\prime \prime}\right)$ is not enough to disentangle what actually comes from the very centre. The extinction might also be a problem.

\subsubsection{Dust continuum emission}

Another possibility is that the continuum comes from thermal dust emission. At millimeter wavelengths we are nearly in the Rayleigh-Jeans domain, and the dust emission is only proportional to the dust temperature. Continuum dust emission is then expected to be quite similar in morphology to the $\mathrm{CO}(3-2)$ emission (e.g. Dumke et al. 1997). Why is this not the case? The difference might be due to the lack of short spacing data, and the filtering out of the diffuse extended continuum emission. Indeed, the continuum is much more sensitive to this problem than the line emission. From the IRAS fluxes, the average temperature of the dust in NGC 1433 can be estimated as $24 \mathrm{~K}$, assuming that the dust opacity has a dependence in frequency of $\gamma^{\beta}$, with $\beta=2$. This is similar to central dust temperatures observed in $\sim 40^{\prime \prime}$ beams with Herschel in star-forming barred galaxies such as NGC 3627 (Hunt et al. 2013, in prep.). From a flux of $0.5 \mathrm{mJy} / \mathrm{beam}$, and assuming the same Draine \& Lee (1984) dust absorption cross section as described in Dumke et al. (1997) for a solar metallicity, we find a molecular gas column density of $N\left(\mathrm{H}_{2}\right)=4.5 \times 10^{22} \mathrm{~cm}^{-2}$, over a beam of $24 \mathrm{pc}$ in size. This is what is expected from a typical giant molecular cloud. In comparison, in the same position, the $\mathrm{CO}(3-2)$ emission is about $4 \mathrm{Jy} \mathrm{km} \mathrm{s}^{-1}$, for a $\mathrm{CO}$ integrated intensity in one beam of $262 \mathrm{~K} \mathrm{~km} \mathrm{~s}^{-1}$, corresponding to $N\left(\mathrm{H}_{2}\right)=6 \times 10^{22} \mathrm{~cm}^{-2}$, with a standard conversion factor of $2.3 \times 10^{20} \mathrm{~cm}^{-2} /\left(\mathrm{K} \mathrm{km} \mathrm{s}^{-1}\right)$ (e.g. Solomon \& Vanden Bout 2005). Considering all the uncertainties, the continuum emission is at the level expected from dust alone. Given that dust emission is only detected at the very centre, it might be possible that this dust is associated with the molecular torus expected to hide the AGN in this Seyfert 2 galaxy. The derived mass of the torus would be $9 \times 10^{5} M_{\odot}$. Since the dust in the torus is certainly warmer than in the disk, this might also explain why the continuum emission is not more extended, as is the $\mathrm{CO}$, in addition to the interferometer's filtering argument explained above. Midinfrared maps with ISO at 7 and $15 \mu \mathrm{m}$ also show a high central concentration, but with low resolution (Roussel et al. 2001).
Only high-resolution observations with ALMA at several different frequencies would be able to settle the origin of the continuum emission and determine whether the AGN is detected directly.

\subsection{CO kinematics: a molecular outflow?}

In a previous paper (Buta et al. 2001), a detailed mass model of NGC 1433 was performed from near-IR photometry and $\mathrm{H} \alpha$ spectroscopy. Rotational and epicyclic frequencies $(\Omega$ and $\kappa$ ) were then derived, and together with the numerical simulations from Buta \& Combes (2000), the predictions of the resonance locations, compared to the observed ring radii, favoured a pattern speed of $23 \mathrm{~km} \mathrm{~s}^{-1} / \mathrm{kpc}$ (or $26 \mathrm{~km} \mathrm{~s}^{-1} / \mathrm{kpc}$ with our slightly different distance adopted). With this pattern speed, there are two inner Lindblad resonances (ILRs) located at 3.6 and $30^{\prime \prime 2}$. The existence of two ILRs weakens the primary bar, and allows the decoupling of a secondary bar, with a higher pattern speed (e.g. Friedli \& Martinet 1993; Buta \& Combes 1996). The nuclear bar produces negative torques on the gas, previously stalled at the nuclear ring, and provides a dynamical way to fuel the nucleus. This process has been simulated in detail in Hunt et al. (2008), and shows how the gas in the nuclear ring progressively flows to the centre, in a spiral structure and in a ring shrinking in radius. It appears that this scenario applies quite well to NGC 1433: its nuclear ring lies between the two ILRs, and the molecular gas morphology reveals an accumulation of the gas at the inner ILR. This configuration strongly suggests that the gas is presently fueling the AGN.

The top panel of Fig. 2 displays the velocity field of the molecular gas. The velocity field is well described by rotation, with the same position angle as the HI velocity field at larger scales (Ryder et al. 1996) and is consistent with the $\mathrm{H} \alpha$ central kinematics (Buta et al. 2001). Superposed on this regular rotation, there are no strong perturbations due to streaming motions in a barred potential, since the major axis of the galaxy is aligned with the minor axis of the primary bar, and also with the nuclear bar (see Fig. 3). The amplitude of the rotation is low but compatible with the observed $\mathrm{H} \alpha$ velocities within $10^{\prime \prime}$ in radius, given the low inclination of $33^{\circ}$. For purposes of comparison, the rotation velocities deduced from the $\mathrm{CO}$ kinematics and the $\mathrm{H} \alpha$ rotation curve are shown together in Fig. 6.

There is, however, a noticeable redshifted perturbation located at the very centre and it extends to the south-west between 0 and $2^{\prime \prime}$ i.e. $100 \mathrm{pc}$ in extent. To better isolate this feature, we plot the position-velocity diagram along the major axis of the galaxy in the top panel of Fig. 7. This slice reveals gas jumping by almost $100 \mathrm{~km} \mathrm{~s}^{-1}$ in projection, at much higher velocity than the rest of the nuclear disk gas. There is also a noticeable blue-shifted counterpart, at a distance from the centre of about $2^{\prime \prime}(100 \mathrm{pc})$ towards the north-west, which is conspicuous in the position-velocity diagram along the minor-axis of the galaxy in the bottom panel of Fig. 7. Both flow components are seen in this direction, while the largest gradient of velocities is along $\mathrm{PA}=135^{\circ}$, which might be the projected direction of the flow.

Another way to compare these peculiar velocities to the rest of the nuclear region in $2 \mathrm{D}$ is to subtract the expected regular velocity field known from the $\mathrm{H} \alpha$ gas in the same region. Figure 6 displays the residuals obtained, relative to the adopted

2 Treuthardt et al. (2008) propose a lower value for the pattern speed in NGC 1433, $18 \mathrm{~km} \mathrm{~s}^{-1} / \mathrm{kpc}$, but their simulation shows a nuclear ring much larger in size than observed. 

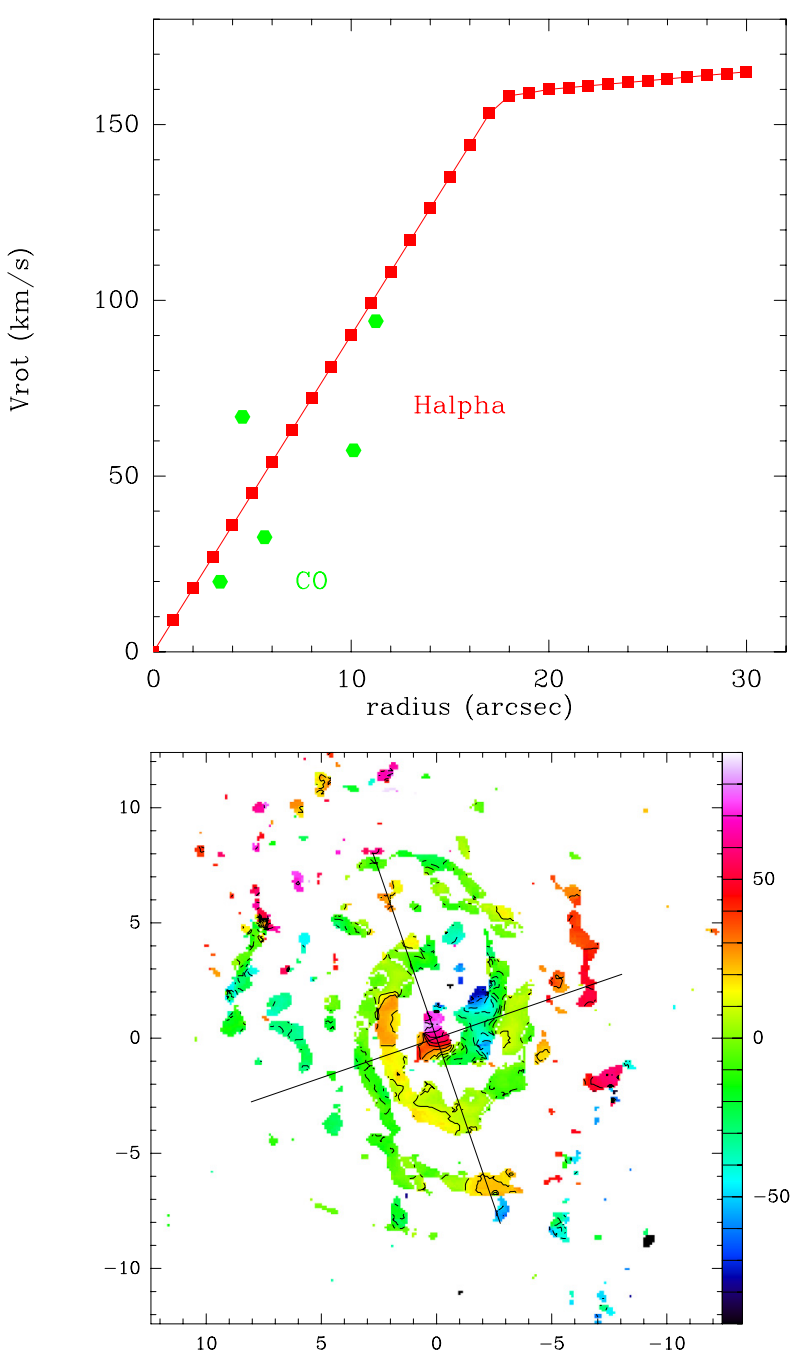

Fig. 6. Top: rotational velocity model adopted for NGC 1433, based on the $\mathrm{H} \alpha$ kinematics (red filled squares) from Buta et al. (2001), compatible with the $\mathrm{CO}$ rotation curve (green filled hexagons). The $\mathrm{CO}$ velocity field is, however, sparsely sampled. Bottom: velocity residuals after subtraction of a regular rotation model, based on the $\mathrm{H} \alpha$ rotation curve above. The map has been recentred on the new adopted centre given in Table 1. The two orthogonal lines indicate the position of the PV diagrams in Fig. 7.

$\mathrm{H} \alpha$ rotation curve, plotted above. The figure shows the ionized gas rotation curve deduced by Buta et al. (2001). The derived $\mathrm{CO}$ velocities, although in sparse regions, are compatible with this adopted rotation curve. The stellar velocity, once corrected for a large asymmetric drift, appears higher (Buta et al. 2001). The gas then does not follow the maximum circular velocity. This might be due to substantial gas turbulence, and/or to an overestimation of the correction of the stellar velocity.

The peculiar velocity of the gas at the nucleus and northwest of the centre is clearly seen in the residuals of Fig. 6. If the gas is in the plane, the deprojected velocity could be as high as $200 \mathrm{~km} \mathrm{~s}^{-1}$, but other orientations with respect to the sky plane are possible. We call $\alpha$ the angle between the outflow direction and the line of sight. The observed velocity in projection is $V_{\text {outflow }} \cos (\alpha)$, and the extent of the flow in the plane of the sky is $R_{\text {outflow }} \sin (\alpha)$. It is likely that $\alpha$ is not close to the extreme values, i.e. zero or 90 degrees, since the observed outflow velocity and the projected size of the outflow are both subtantial,
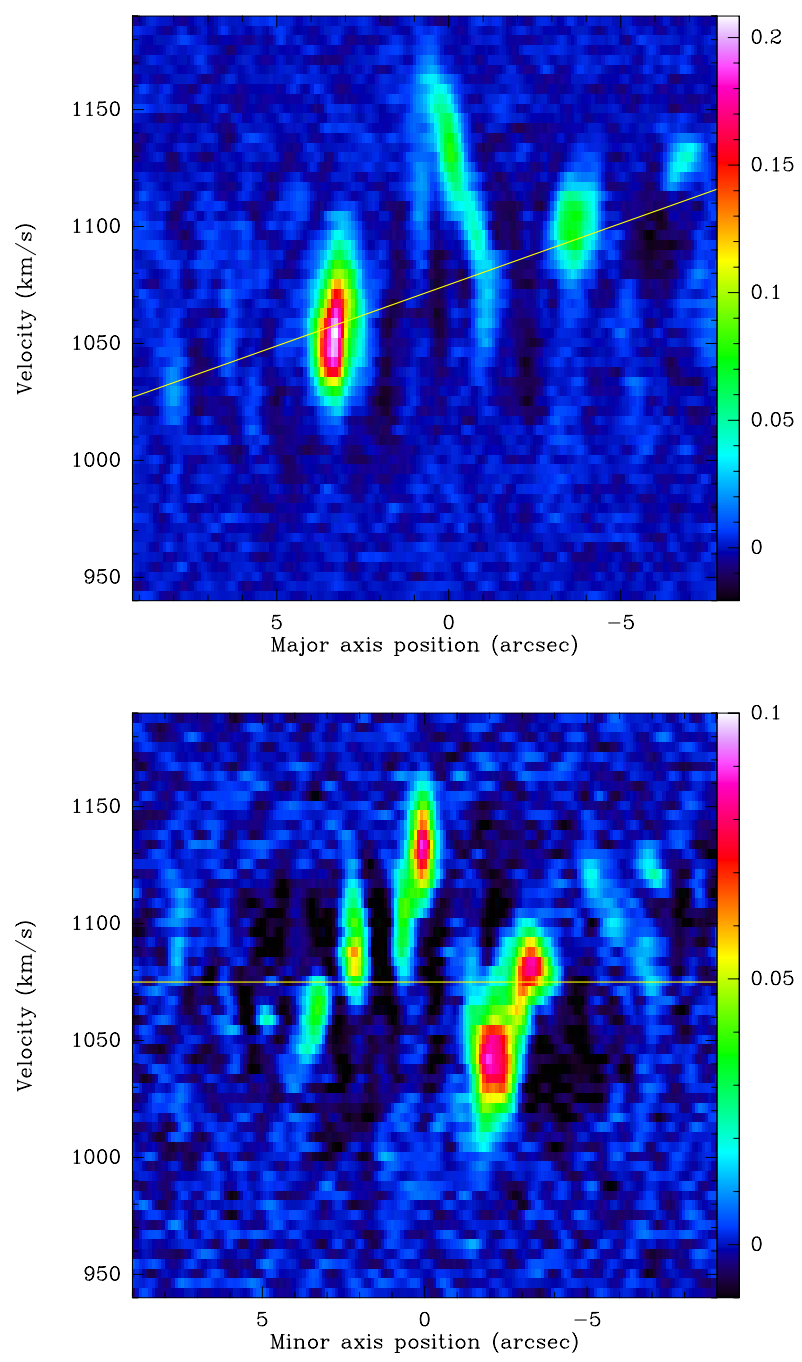

Fig. 7. Top: position-velocity diagram along the major axis of PA = $199^{\circ}$ (east is at left). The central outflow is clearly visible superposed on the smooth rotational velocity gradient (underlined by the yellow line, corresponding to the rotation curve of Fig. 6). Bottom: positionvelocity diagram, along the minor axis, of $\mathrm{PA}=109^{\circ}$ (east is at left). The two components (red and blue) of the outflow are visible, along a slice, where the velocity should be equal to the systemic one (yellow line).

i.e. $\sim 100 \mathrm{~km} \mathrm{~s}^{-1}$ and $\sim 100 \mathrm{pc}$, respectively. This means that $\tan (\alpha)$ is of the order of 1 . The flow is aligned roughly with the minor axis, and if it is orthogonal to the plane, $\tan (\alpha)=0.6$. We think, however, that the outflow is not orthogonal, since we are seing the galaxy inclined by $33^{\circ}$ on the sky, and the near side is the NW, from the winding sense of the spiral arms, assumed trailing. The outflow cannot be exactly perpendicular to the disk, unless the blue and red regions are inverted. The flow must at least be inclined by an angle $>33^{\circ}$ from the normal to the plane. Conservatively, the outflow velocity probably lies between $100 \mathrm{~km} \mathrm{~s}^{-1}$ and $200 \mathrm{~km} \mathrm{~s}^{-1}$.

This high-velocity gas is also noticeable in the total spectrum, obtained by summing the signal over the field of view, as in Fig. 8. A Gaussian decomposition in three components has been performed on the spectrum, and the results are displayed in Table 3 . The high-velocity red component represents nearly $5 \%$ of the total. The blue-velocity counterpart is diluted in the normal rotational component $\mathrm{Cl}$ (part of the two-horn profile characteristic of rotation). 
Table 3. $\mathrm{CO}(3-2)$ line fluxes, after primary beam correction.

\begin{tabular}{lcccc}
\hline \hline Line & $\begin{array}{c}S_{\mathrm{CO}} \\
\mathrm{Jy} \mathrm{km} \mathrm{s}^{-1}\end{array}$ & $\begin{array}{c}V_{\text {hel }} \\
\mathrm{km} \mathrm{s}^{-1}\end{array}$ & $\begin{array}{c}\Delta V^{a} \\
\mathrm{~km} \mathrm{~s}^{-1}\end{array}$ & $\begin{array}{c}\text { Peak flux } \\
\text { Jy }\end{array}$ \\
\hline Total & $234 \pm 1$ & $1073.1 \pm 0.3$ & $85.3 \pm 0.7$ & 2.58 \\
\hline $\mathrm{C} 1$ & $103 \pm 2$ & $1040.0 \pm 0.4$ & $46 . \pm 1$ & 2.1 \\
$\mathrm{C} 2$ & $105 \pm 4$ & $1089.1 \pm 0.2$ & $30 . \pm 1$ & 3.3 \\
$\mathrm{C} 3$ & $26 \pm 3$ & $1123.0 \pm 4.0$ & $59 . \pm 5$ & 0.4 \\
\hline Blue $^{b}$ & $6.0 \pm 0.1$ & $1018.7 \pm 0.6$ & $61 . \pm 1$ & 0.09 \\
Red $^{b}$ & $10.1 \pm 0.1$ & $1138.2 \pm 0.3$ & $56 . \pm 0.7$ & 0.17 \\
\hline
\end{tabular}

Notes. Total $=$ Gaussian fit, assuming only one component, $\mathrm{C} 1 / \mathrm{C} 1 / \mathrm{C} 3$ represent three velocity-component decomposition. ${ }^{(a)}$ Full Width at Half Maximum (FWHM). ${ }^{(b)}$ Fits for the blue and red components of the outflow, summed over a region 0 '. $^{\prime} \times 1 \times 1$ '.2 each (cf Fig. 9).

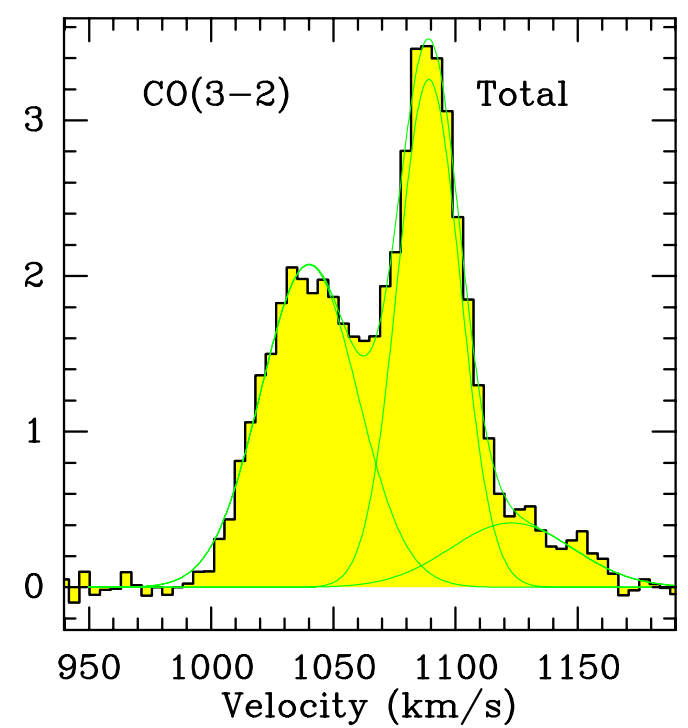

Fig. 8. Total $\mathrm{CO}(3-2)$ spectrum, integrated over the observed map, with a FOV of $18^{\prime \prime}$, after correction for primary beam attenuation. The vertical scale is in Jy. The green line is the result of the Gaussian fit with three velocity components; see Table 3.

Finally, the high-velocity red component is best located in the map through the individual spectra of Fig. 9, where all spectra are shown within a radius of 2 '.5. Although in principle, this high-velocity gas could be inflowing as well as outflowing, we consider inflow to be unlikely. Indeed, there is no other signature of violent perturbation due to a companion nearby, and if gas were slowly accreted to fuel the AGN, it would first have settled into the rotational frame at much larger radii than the last $100 \mathrm{pc}$.

To better estimate the quantity of gas in the outflow, we have summed the CO flux within two regions of sizes $0 . ' 7 \times 1$.' 2 , centred on the red and blue outflow regions, taking into account the primary beam correction (cf Fig. 9). The results are given in Table 3. Assuming the standard CO-to- $\mathrm{H}_{2}$ conversion factor (see next section), we derive molecular masses of $1.3 \times 10^{6}$ and $2.3 \times 10^{6} M_{\odot}$ for the blue and red velocity components, respectively.

Is the outflow also detected in the ionized gas? There is no outflow detected in X-rays, but there is not enough spatial resolution to see it anyway. In $\mathrm{H} \alpha$ maps and spectroscopy, it is hard to reach a conclusion, even from the best velocity field obtained from Fabry-Perot interferometry by Buta (1986). In his
Fig. 8, we can see a quite perturbed velocity field inside the central $20^{\prime \prime}$, which may reflect steep gradients. However, the spatial resolution is only $2^{\prime \prime}$, while the projected distance between our red and blue outflow peak components is roughly the same. An outflow of ionized gas is, however, compatible with the data. The non-detection of ionized gas outflow in galaxies showing a molecular outflow is also found in other compact systems like NGC 1377 (Aalto et al. 2012). A comparison with other molecular outflows will be discussed in Sect. 4.

\section{4. $\mathrm{CO}$ luminosity, $\mathrm{H}_{2}$ mass, and $\mathrm{HCO}^{+} / \mathrm{HCN}$ upper limits}

Figure 8 displays the total $\mathrm{CO}(3-2)$ spectrum, integrated over the entire observed map after correction for primary beam attenuation. When integrated over the line $\left(F W H M=85.3 \mathrm{~km} \mathrm{~s}^{-1}\right)$, the integrated emission is $234 \pm 1 \mathrm{Jy} \mathrm{km} \mathrm{s}^{-1}$. Towards the central position, Bajaja et al. (1995) found a $\mathrm{CO}(1-0)$ spectrum peaking at $T_{A}^{*}=48 \mathrm{mK}$, with $F W H M=168 \mathrm{~km} \mathrm{~s}^{-1}$, yielding a total integrated flux of $193 \mathrm{Jy} \mathrm{km} \mathrm{s}^{-1}$, in a beam of $43^{\prime \prime}$. Unfortunately, no $\mathrm{CO}(2-1)$ spectra have been reported. We can, however, remark that the $\mathrm{CO}(1-0)$ flux corresponds to a larger region than the one observed here (as witnessed by the broader linewidth), and our $\mathrm{CO}(3-2)$ flux is an upper limit of the expected $\mathrm{CO}(1-0)$ flux in a $18^{\prime \prime}$ beam, since at low $J$ the flux increases with the $J$ level. We can therefore safely conclude that the $\mathrm{CO}(3-2) / \mathrm{CO}(1-0)$ ratio must be significantly larger than 2 in flux density units: the $\mathrm{CO}$ gas is relatively excited, meaning that the average density is at least of the order of $10^{4} \mathrm{~cm}^{-3}$. Assuming a $\mathrm{CO}(3-2) / \mathrm{CO}(1-0)$ flux ratio of $\sim 5$, similar to that observed in the star-forming nuclei of nearby galaxies (e.g. Matsushita et al. 2004; Boone et al. 2011), the CO detected inside our primary beam, at the distance of $9.9 \mathrm{Mpc}$, corresponds to a molecular mass $M\left(\mathrm{H}_{2}\right)=5.2 \times 10^{7} M_{\odot}$, with the standard CO-to- $\mathrm{H}_{2}$ conversion factor of $2.3 \times 10^{20} \mathrm{~cm}^{-2} /\left(\mathrm{K} \mathrm{km} \mathrm{s}^{-1}\right)$. By comparison, Bajaja et al. (1995) find in their central 43" beam a mass of $1.8 \times 10^{8} M_{\odot}$, covering an area 5.7 times larger.

As far as the $\mathrm{CO}$ outflow is concerned, the use of any CO-to- $\mathrm{H}_{2}$ conversion factor is uncertain. We then try to get a strict minimum of the mass in using the hypothesis of optically thin emission. Within this hypothesis, we can write the column density of $\mathrm{CO}$ in the upper state of the (3-2) transition as:

$N_{J=3}=6.4 \times 10^{13} I_{\mathrm{CO}(3-2)}$,

where $I_{\mathrm{CO}(3-2)}$ is the integrated (3-2) emission in $\mathrm{K} \mathrm{km} \mathrm{s}^{-1}$. The total $\mathrm{CO}$ column density is then obtained, using the ratio

$N_{J=3} / N_{\mathrm{CO}}=\frac{5}{Q} \exp \left(-E_{J=3} / k T_{\mathrm{ex}}\right)$,

where $E_{J=3}$ is the energy in the upper level of the (3-2) transition, Q the partition function $=0.36 T_{\mathrm{ex}}$, and $T_{\mathrm{ex}}$ the excitation temperature assumed constant over all $J$ levels. The $N\left(\mathrm{H}_{2}\right)$ column density is then derived, assuming a $\mathrm{CO}$ abundance of $6 \times 10^{-5}$ (e.g. Glover \& Mac Low 2011). Comparing the $N\left(\mathrm{H}_{2}\right)$ values obtained within the optically thick hypothesis, and the use of the standard conversion factor, we found column densities lower by factors of 27,71 , and 83 when $T_{\mathrm{ex}}=10$, 20 , and $30 \mathrm{~K}$, respectively. Over the red outflow region that is as large as 3-4 beams, we found $N\left(\mathrm{H}_{2}\right) \sim 5 \times 10^{22} \mathrm{~cm}^{-2}$, while the optically thin hypothesis will lead to values as low as $6 \times 10^{20} \mathrm{~cm}^{-2}$. We estimate that values this low are not realistic, however, since the mean volumetric density over the region will be $\sim 1 \mathrm{~cm}^{-3}$, and not $100 \mathrm{~cm}^{-3}$, the minimum required 


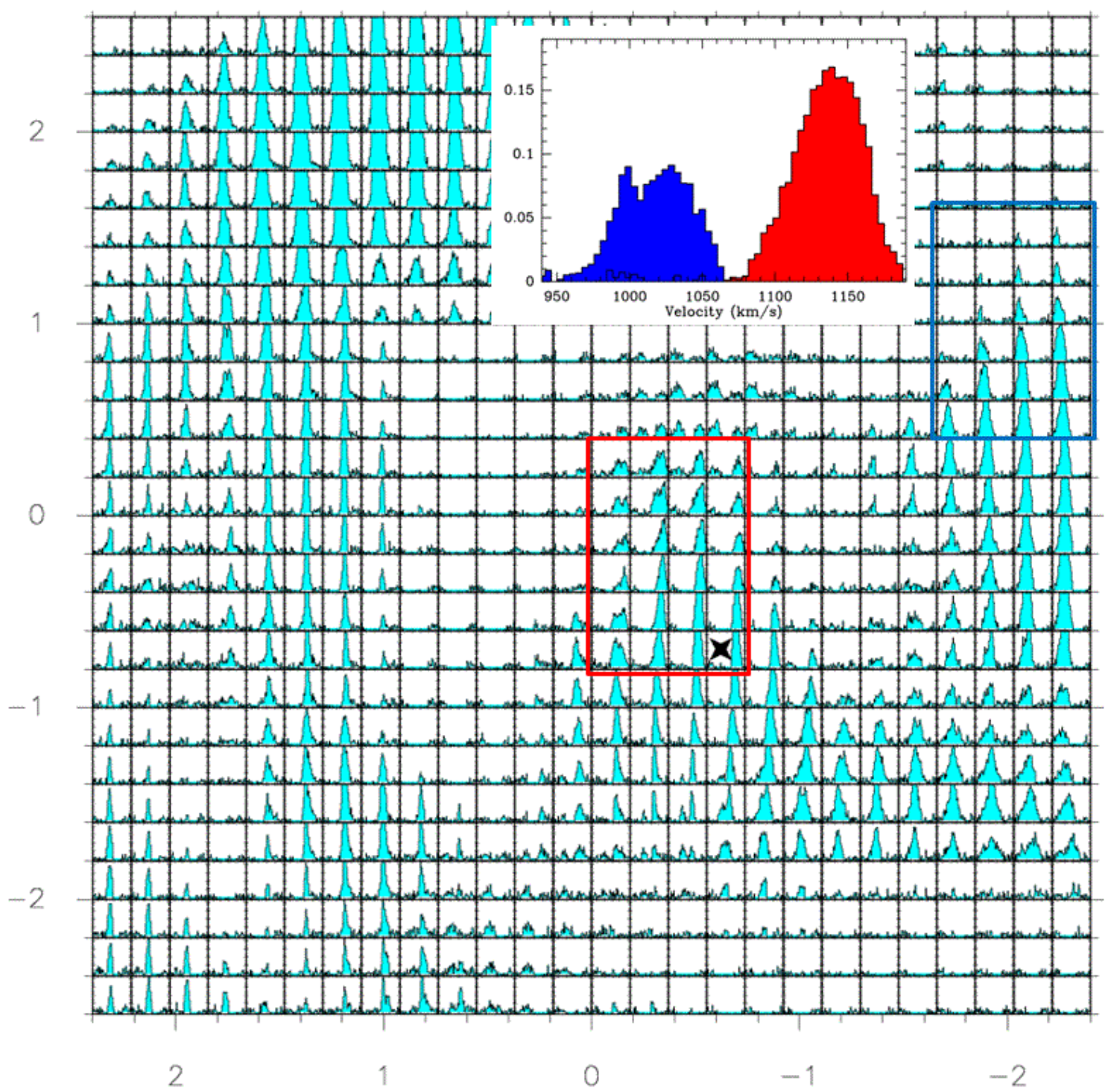

Fig. 9. $\mathrm{CO}(3-2)$ spectra within 2 '. 5 of the centre. The velocity scale is from 960 to $1190 \mathrm{~km} \mathrm{~s}^{-1}$ (corresponding to a range from -115 to $115 \mathrm{~km} \mathrm{~s}^{-1}$ with respect to the systemic velocity). The vertical scale is from 1 to $40 \mathrm{mJy}$. The new adopted centre is marked with a black star (the map coordinates are with respect to the phase centre). The red velocity component can be seen at the new centre and just above, and the blue velocity component is centred at $(-2,0.5)$. The two regions of $0.7 \times 1 . \prime 2$ each, selected to integrate the outflow mass in Table 3 , are indicated by red and blue rectangles, respectively. The corresponding integrated spectra are plotted in the insert (scale in Jy).

to excite $\mathrm{CO}$ emission. We note that the mean $\mathrm{CO}(3-2)$ brightness temperature observed within the flow region if $3 \mathrm{~K}$, so that the surface filling factor of the molecular component cannot be much smaller than 0.1 . Since the critical density of the $\mathrm{CO}(3-2)$ line is $10^{5} \mathrm{~cm}^{-3}$, the optically thin hypothesis is very unlikely to provide any emission, even taking into account the surface filling factor.

Finally, our simultaneous observations of $\mathrm{HCO}^{+}(4-3)$ and HCN(4-3) yielded only negative results. We can better derive significant upper limits towards the $\mathrm{CO}$ emission maxima. Over the whole map, there were 160 pixels (equivalent to 8 beams) with $\mathrm{CO}(3-2)$ emission larger than 60 times the $3 \sigma$ upper limits in $\mathrm{HCO}^{+}(4-3)$ and $\mathrm{HCN}(4-3)$, assuming the same linewidth. In all $\mathrm{CO}$ maxima, an intensity ratio between $\mathrm{CO}$ and the highdensity tracers $>60$ means that the average density of the gas in the multiple-arm flocculent spiral is not high. The critical density to excite the $\mathrm{HCO}^{+}(4-3)$ and $\mathrm{HCN}(4-3)$ molecular lines is at least $10^{7} \mathrm{~cm}^{-3}$.

\section{Discussion and summary}

We have presented our first ALMA results for a Seyfert 2 galaxy from our extended NUGA sample, NGC 1433. The observations in $\mathrm{CO}(3-2)$ allow us to reach an unprecedented spatial resolution of $24 \mathrm{pc}$, even with the limited Cycle 0 capabilities.

The morphology of the $\mathrm{CO}$ emission comes as a surprise. Although the Seyfert 2-type would suggest the presence of a thick obscuring component in front of the nucleus, there is no large concentration of molecular gas in the centre, but instead a widely distributed multiple-arm spiral of $\mathrm{CO}$ emission all over the nuclear ring region. The dense gas tracers $\mathrm{HCO}^{+}$and $\mathrm{HCN}$ remain undetected, confirming the absence of very dense gas (density larger than $10^{7} \mathrm{~cm}^{-3}$ ).

Although infrared images reveal the presence of a stellar nuclear bar inside the nuclear ring (of radius $0.5 \mathrm{kpc}$ ), located near the inner Lindblad resonances (e.g. Buta et al. 2001), the 
gas does not follow the nuclear bar. Instead the gas appears to flow inward and to partly accumulate in a ring-like structure at a radius $\sim 200 \mathrm{pc}$, which coincides with the inner ILR (IILR) as computed by Buta et al. (2001). This is indeed expected at some epochs of self-consistent $N$-body+hydro simulations, when the gas enters an inflowing phase inside two ILRs (e.g. Hunt et al. 2008). The gas is not stalled in this pseudo-ring, but continues to flow in towards the very centre.

The kinematics of the $\mathrm{CO}$ emission are dominated by a regular rotational velocity field, with only slight perturbations from the multiple-arm spiral. No strong streaming motion is imprinted on these kinematics by the primary and nuclear bars, since their axes coincide with the galaxy major axis. Additionally, two peculiar features appear at high velocity, a red-shifted component towards the centre within $100 \mathrm{pc}$, and a blue-shifted counterpart at $2^{\prime \prime}(100 \mathrm{pc})$ from the centre. The amplitude of these components is up to about $100 \mathrm{~km} \mathrm{~s}^{-1}$ in projection $\left(\sim 200 \mathrm{~km} \mathrm{~s}^{-1}\right.$ if in the galaxy plane). Given their location near the nucleus, we tentatively interpret these high-velocity features as the two sides of an outflow. Globally, these features represent as much as $\sim 7 \%$ of the total molecular emission in the nuclear ring region, i.e., $3.6 \times 10^{6} M_{\odot}$.

It is not likely that these peculiar high-velocity features reflect strong streaming motions due to a dynamical perturbation, since there is no perturbation of this kind in the centre. The gas does not follow the nuclear bar, which is rather weak. Is a central mass able to generate such a high rotation in the centre? Considering that the blue and red components are separated in projection along the minor axis by $2^{\prime \prime}=100 \mathrm{pc}$, or about $120 \mathrm{pc}$ in the plane of the galaxy, a massive black hole located in the centre, at $R=60 \mathrm{pc}$ from each component, should have a mass of at least $M_{\mathrm{BH}}=V^{2} R / \mathrm{G}$ for the rotational velocity in the galaxy plane $V=200 \mathrm{~km} \mathrm{~s}^{-1}$, or $M_{\mathrm{BH}}=5.6 \times 10^{8} M_{\odot}$. This would make NGC 1433 a strong outlier to the $M_{\mathrm{BH}}-\sigma$ relation; indeed from the bulge mass, we would expect the $\mathrm{BH}$ mass to be $5 \times 10^{6} M_{\odot}$, e.g., Buta (1986). In any case, for gas rotating in circular motion within the sphere of influence of the black hole, the velocity maxima should appear on the major axis and disappear on the minor axis, contrary to what is observed here. Another solution would be to assume the existence of a minipolar disk with completely different orientation to the main disk, and almost edge-on, but no galaxy interaction or accretion event supports this hypothesis.

The origin of the outflow might be related to star formation, which is concentrated in the nuclear ring region. The SFR can be estimated from the far-IR luminosity as calibrated by Kennicutt (1998). From the IRAS fluxes, the far-IR luminosity is $1.3 \times 10^{9} L_{\odot}$ (Table 1 ), and the SFR equals $0.2 M_{\odot} /$ yr. From the $\mathrm{H} \alpha$ luminosity, measured at $3.7 \times 10^{40} \mathrm{erg} / \mathrm{s}$ by Hameed $\&$ Devereux (2005), we can also deduce from Kennicutt's calibration a $S F R=0.29 M_{\odot} / \mathrm{yr}$, which is compatible.

The order of magnitude of the mass outflow rate can be computed, using our estimates for the molecular mass in the high-velocity components (Table 3 ), as $M=3.6 \times 10^{6} M_{\odot}$. This mass was obtained using the standard $\mathrm{CO}-$ to- $\mathrm{H}_{2}$ conversion factor, since there is no reason a priori to adopt the lower factor applying to ultra-luminous galaxies. Cicone et al. (2012) show in Mrk231 that the molecular gas of the galaxy and the outflowing gas share the same excitation. However, this mass could be an upper limit if the flow is made of more diffuse gas. Since each high-velocity component has a projected radial extent from the centre of $d=1^{\prime \prime} \sim 50 \mathrm{pc}$, and moves at a projected velocity of $v=100 \mathrm{~km} \mathrm{~s}^{-1}$, the flow rate is of the order of $\mathrm{d} M / \mathrm{d} t \sim(M v / d) \tan \alpha=7 \tan \alpha M_{\odot} / \mathrm{yr}$, with $\alpha$ being the angle between the outflow and the line of sight.

Although this estimate is uncertain by a factor of a few, given the unknown $\alpha$, it is about 40 times higher than the SFR; since galactic winds due to starbursts correspond in general to mass outflows of the same order as the SFR (e.g. Veilleux et al. 2005), we conclude that the outflow is probably not due to star formation alone, and is at least helped by the AGN. We note that starburst winds are generally observed in galaxies with SFR larger than $5 M_{\odot} / y r$, and SFR surface densities larger than $10^{-3} M_{\odot} / \mathrm{yr} / \mathrm{kpc}^{2}$. The galaxy NGC 1433 has a low total SFR $0.2 M_{\odot} / \mathrm{yr}$; however, its SFR surface density is $0.34 M_{\odot} / \mathrm{yr} / \mathrm{kpc}^{2}$ if we assume that the whole SFR is confined to the nuclear disk of $9^{\prime \prime}$ radius. The SFR surface density would therefore be enough to drive a wind, although (as noted above) the mass loading factor expected for this type of wind would still be considerably lower than what we observe in NGC 1433.

The kinetic luminosity of the flow can be estimated as $L_{\mathrm{kin}}=$ $0.5 \mathrm{~d} M / \mathrm{d} t v^{2}=2.3 \tan \alpha\left(1+\tan ^{2} \alpha\right) \times 10^{40} \mathrm{erg} / \mathrm{s}$. The luminosity of the AGN can be estimated at various wavelengths. Although the X-ray point source is weak, $1.7 \times 10^{39} \mathrm{erg} / \mathrm{s}$ over $0.3-8 \mathrm{kev}$ (Liu \& Bregman 2005), we can derive a bolometric luminosity of the AGN from optical and near-IR magnitudes in the central aperture (Buta et al. 2001) of $1.3 \times 10^{43} \mathrm{erg} / \mathrm{s}$. From the expected BH mass of $5 \times 10^{6} M_{\odot}$, if NGC 1433 is on the $M_{\mathrm{BH}}-\sigma$ relation, the Eddington luminosity is $6.3 \times 10^{44} \mathrm{erg} / \mathrm{s}$. The kinetic luminosity of the outflow is low with respect to the bolometric luminosity of the AGN, making it plausible that the latter is able to power the wind.

The momentum flux of the outflow, computed by $\mathrm{d} M / \mathrm{d} t v$ is, however, too large compared to that provided by the AGN photons $L_{\mathrm{AGN}} / c$, by a factor of $2000 \tan \alpha / \cos \alpha$. Although the momentum can be boosted in case of energy-conserved wind by factors up to 50 (e.g. Faucher-Giguère \& Quataert 2012), it is more likely that the AGN contributes to drive the outflow not by its radiation pressure, but through its radio jets. From the central $1.4 \mathrm{GHz}$ power of $3.4 \mathrm{mJy}$ detected by Ryder et al. (1996), we can estimate the jet power from the formula proposed by Birzan et al. (2008, their Eq. (16)): $P_{\text {jet }}=2 \times 10^{42} \mathrm{erg} / \mathrm{s}$. Since this power is about two orders of magnitudes higher than the kinetic luminosity of the outflow, the jet is amply able to drive the flow, even with low coupling. The jet interaction with the interstellar medium has been simulated by Wagner et al. (2012) who show that the jet is able to drive a flow efficiently as soon as the Eddington ratio of the jet $P_{\text {jet }} / L_{\text {Edd }}$ is larger than $10^{-4}$. In NGC 1433, this ratio is about $3.2 \times 10^{-3}$.

The molecular outflow in NGC 1433 is one of only a few discovered recently occuring in low-star forming galaxies with relatively weak AGN where the flow might be driven by both the starburst and the radio jets. The LINER NGC 6764 has $4.3 \times 10^{6} M_{\odot}$ of molecular gas driven out with a velocity of about $100 \mathrm{~km} \mathrm{~s}^{-1}$ (Leon et al. 2007). The flow projects to larger distances than in NGC 1433, and might be more evolved. The outflow rate is lower, of the order of $1 M_{\odot} / \mathrm{yr}$. The galaxy NGC 1266 is also a LINER and has the highest flow rate of $13 M_{\odot} / \mathrm{yr}$, with $2.4 \times 10^{7} M_{\odot}$ of molecular gas driven with $V=177 \mathrm{~km} \mathrm{~s}^{-1}$ (Alatalo et al. 2011). A third LINER with total SFR of $\sim 1 M_{\odot} / \mathrm{yr}$, NGC 1377 has an outflow rate of $8 M_{\odot} / \mathrm{yr}$, and an outflowing mass of $1.1 \times 10^{7} M_{\odot}$ at $V=140 \mathrm{~km} \mathrm{~s}^{-1}$ (Aalto et al. 2012). All these galaxies have star formation playing a role in the outflow, but the properties of the flow require the contribution of the AGN through the entrainement of its radio jets. This is the most needed for NGC 1433, which has the lowest SFR of all. 
This tentative detection of a molecular gas outflow triggered essentially by the AGN, should be confirmed by higherresolution ALMA observations. The detection of a radio continuum component at the very centre, which might be due to thermal dust emission from a molecular torus, also deserves a higher resolution study.

Acknowledgements. We warmly thank the referee for constructive comments and suggestions. The ALMA staff in Chile and ARC-people at IRAM are gratefully acknowledged for their help in the data reduction. We particularly thank Gaelle Dumas and Philippe Salomé for their useful advice. This paper makes use of the following ALMA data: ADS/JAO.ALMA\#2011.0.00208.S. ALMA is a partnership of ESO (representing its member states), NSF (USA) and NINS (Japan), together with NRC (Canada) and NSC and ASIAA (Taiwan), in cooperation with the Republic of Chile. The Joint ALMA Observatory is operated by ESO, AUI/NRAO and NAOJ. The National Radio Astronomy Observatory is a facility of the National Science Foundation operated under cooperative agreement by Associated Universities, Inc. We used observations made with the NASA/ESA Hubble Space Telescope, and obtained from the Hubble Legacy Archive, which is a collaboration between the Space Telescope Science Institute (STScI/NASA), the Space Telescope European Coordinating Facility (ST-ECF/ESA), and the Canadian Astronomy Data Centre (CADC/NRC/CSA). F.C. acknowledges the European Research Council for the Advanced Grant Program Num 267399-Momentum. I.M. acknowledges financial support from the Spanish grant AYA2010-15169 and from the Junta de Andalucia through TIC-114 and the Excellence Project P08-TIC-03531. We made use of the NASA/IPAC Extragalactic Database (NED), and of the HyperLeda database.

\section{References}

Aalto, S., Muller, S., Sakamoto, K., et al. 2012, A\&A, 546, A68 Alatalo, K., Blitz, L., Young, L. M., et al., 2011, ApJ, 735, 88 Bajaja, E., Wielebinski, R., Reuter, H. P., et al. 1995, A\&AS, 114, 147 Birzan, L., McNamara, B. R., Nulsen, P. E. J., et al. 2008, ApJ, 686, 859 Boone, F., Garcia-Burillo, S., Combes, F., et al. 2011, A\&A, 525, A18 Buta, R. 1986, ApJS, 61, 631

Buta, R., \& Combes, F. 1996, Fund. Cosm. Phys., 17, 95

Buta, R., \& Combes, F. 2000, in Dynamics of Galaxies: From the Early Universe to the Present, eds. F. Combes, G. A. Mamon, \& V. Charmandaris, ASP Conf. Ser., 197, 11

Buta, R., Ryder, S. D., Madsen, G. J., et al. 2001, AJ, 121, 225

Casasola, V., Combes, F., \& Garcia-Burillo, S. 2008, A\&A, 490, 61

Casasola, V., Hunt, L. K., Combes, F., Garcia-Burillo, S., \& Neri, R. 2011, A\&A, 527, A92

Chung, A., Yun, M. S., Naraynan, G., Heyer, M., \& Erickson, N. R. 2011, ApJ, 732, L15

Cicone, C., Feruglio, C., Maiolino, R. et al. 2012, A\&A, 543, A99

Coil, A. L., Weiner, B. J., Holz, D. E., et al. 2011, ApJ, 743, 46

Combes, F. 2003 ASP Conf. Ser., 290, 411

Combes, F. 2006, in Astrophysics Update 2 (Springer), 159

Comeron, S., Knapen, J. H., Beckman, J. E., et al. 2010, MNRAS, 402, 2462

Croton, D. J., Springel, V., White, S. D. M., et al. 2006, MNRAS, 365, 11

Dasyra, K., \& Combes, F. 2012, A\&A, 541, L7

Di Matteo, T., Colberg, J., Springel, V., Hernquist, L., \& Sijacki, D. 2008, ApJ, 676, 33

Draine, B. T., \& Lee, H. M. 1984, ApJ, 285, 89

Dumke, M., Braine, J., Krause, M., et al. 1997, A\&A, 325, 124

Faucher-Giguère, C.-A., \& Quataert, E. 2012, MNRAS, 425, 605

Feruglio, C., Maiolino, R., \& Piconcelli, E., et al. 2010, A\&A, 518, L155
Feruglio, C., Fiore, F., Piconcelli, E., et al. 2013, A\&A, 549, A51

Fischer, J., Sturm, E., Gonzalez-Alfonso, E., et al. 2010, A\&A, 518, L41

Friedli, D., \& Martinet, L. 1993, A\&A, 277, 27

Galliano, E., \& Aloin, D. 2002, A\&A, 393, 43

Garcìa-Burillo, S., Fernandez-Garcia, S., Combes, F., et al. 2009, A\&A, 496, 85

Garcìa-Burillo, S., Usero, A., Fuente, A., et al. 2010, A\&A, 519, A2

Glover, S. C. O., \& Mac Low, M.-M. 2011, MNRAS, 412, 337

Guilloteau, S., \& Lucas, R. 2000, in Imaging at Radio through Submillimeter Wavelengths, ASP Conf. Proc., 217, 299

Hameed, S., \& Devereux, N. 2005, AJ, 129, 2597

Harnett, J. I. 1987, MNRAS, 227, 887

Ho, L. C., Li, Z.-Y., Barth, A. J., et al. 2011, ApJS, 197, 21

Hopkins, P. F., \& Quataert, E. 2010, MNRAS, 407, 1529

Hopkins, P., Hernquist, L., Cox, T. J., et al. 2006, ApJS, 163, 1

Hunt, L. K., \& Malkan, M. A. 1999, ApJ, 516, 660

Hunt, L. K., Combes, F., Garcia-Burillo, S., et al. 2008, A\&A, 482, 133

Jogee, S. 2006, Lect. Notes Phys., 693, 143

Jungwiert, B., Combes, F., \& Axon, D. J. 1997, A\&AS, 125, 479

Kennicutt, R. C. 1998, ApJ, 498, 541

Kilborn, V. A., Koribalsi, B. S., Forbes, D. A., et al. 2005, MNRAS, 356, 77

Kormendy, J., \& Ho, L. C. 2013, ARA\&A, in press [arXiv: 1308.6483]

Krips, M., Martin, S., Eckart, A., et al. 2011, ApJ, 736, 37

Leon, S., Eckart, A., Laine, S., et al. 2007, A\&A, 473, 747

Lindt-Krieg, E., Eckart, A., Neri, R., et al. 2008, A\&A, 479, 377

Liu, J.-F., \& Bregman, J. N. 2005, ApJS, 157, 59

Malkan, M., Gorjian, V., \& Tam, R. 1998, ApJS, 117, 25

Maoz, D., Barth, A. J., Sternberg, A., et al. 1996, AJ, 111, 2248

Matsushita, S., Sakamoto, K., Kuo, C.-Y., et al.. 2004, ApJ, 616, L55

McMullin, J. P., Waters, B., Schiebel, D., Young, W., \& Golap, K. 2007, in Astronomical Data Analysis Software and Systems XVI, ASP Conf. Ser. 376,127

Müller Snchez, F., Davies, R. I., Genzel, R., et al. 2009, ApJ, 691, 749

Nesvadba, N. P. H., Polletta, M., Lehnert, M. D., et al. 2011, MNRAS, 415, 2359

Peeples, M. S., \& Martini, P. 2006, ApJ, 652, 1097

Perez-Beaupuits, J., Wada, K., \& Spaans, M. 2011, ApjJ, 730, 48

Piner, B. G., Stone, J. M., \& Teuben, P. J. 1995, ApJ, 449, 508

Regan, M. W., \& Teuben, P. J. 2004, ApJ, 600, 595

Riffel, R. A., \& Storchi-Bergmann, T. 2011, MNRAS, 411, 469

Roussel, H., Vigroux, L., Bosma, A., et al. 2001, A\&A, 369, 473

Rupke, D. S., Veilleux, S., \& Sanders, D. B. 2005, ApJ, 632, 751

Ryder, S. D., Buta, R. J., \& Toledo, H. 1996, ApJ, 460, 665

Sadler, E. M., Slee, O. B., Reynolds, J. E., \& Roy, A. L. 1995, MNRAS, 276, 1373

Sakamoto, K, Aalto, S., Wilner, D., et al. 2009, ApJ, 700, L104

Schinnerer, E., Eckart, A., Tacconi, L. J., Genzel, R., \& Downes, D. 2000, ApJ, 533,850

Solomon, P. M., \& Vanden Bout, P. A. 2005, ARA\&A, 43, 677

Sosa-Brito, R. M., Tacconi-Garman, L. E., Lehnert, M. D., \& Gallimore, J. F. 2001, ApJS, 136, 61

Spoon, H. W. W., Farrah, D., Lebouteiller, V., et al., 2013, ApJ, submitted [arXiv: 1307.6224]

Stil, J. M., Krause, M., Beck, R., \& Taylor, A. R. 2009, ApJ, 693, 1392

Sturm, E., Gonzalez-Alfonso, E., Veilleux, S., et al. 2011, ApJ, 733, L16

Treuthardt, P., Salo, H., Rautiainen, P., \& Buta, R. 2008, AJ, 136, 300

Ulvestad, J. S., \& Ho, L. C. 2001, ApJ, 558, 561

Veilleux, S., Cecil, G., \& Bland-Hawthorn, J. 2005, ARA\&A, 43, 769

Veilleux, S., Melendez, M., Sturm, E. et al. 2013, ApJ, submitted [arXiv: 1308.3139]

Veron-Cetty, M. P., \& Veron, P. 1986, A\&AS, 66, 335

Wagner, A. Y., Bicknell, G. V., \& Umemura, M. 2012, ApJ, 757, 136 